\title{
Sensorimotor Cortical Neuroplasticity in the Early Stage of Bell's Palsy
}

\author{
Wenwen Song, ${ }^{1,2}$ Minhui Dai, ${ }^{1}$ Lihua Xuan, ${ }^{1}$ Zhijian Cao, ${ }^{1}$ Sisi Zhou, ${ }^{1}$ Courtney Lang, \\ Kun Lv, ${ }^{1}$ Maosheng Xu, ${ }^{1}$ and Jian Kong ${ }^{2}$ \\ ${ }^{1}$ The First Affiliated Hospital of Zhejiang Chinese Medical University, Hangzhou, China \\ ${ }^{2}$ Department of Psychiatry, Massachusetts General Hospital and Harvard Medical School, Charlestown, MA 02129, USA
}

Correspondence should be addressed to Maosheng Xu; xums166@zcmu.edu.cn and Jian Kong; jkong2@mgh.harvard.edu

Received 16 August 2016; Revised 22 December 2016; Accepted 5 January 2017; Published 19 February 2017

Academic Editor: Lin Xu

Copyright (C) 2017 Wenwen Song et al. This is an open access article distributed under the Creative Commons Attribution License, which permits unrestricted use, distribution, and reproduction in any medium, provided the original work is properly cited.

Neuroplasticity is a common phenomenon in the human brain following nerve injury. It is defined as the brain's ability to reorganize by creating new neural pathways in order to adapt to change. Here, we use task-related and resting-state fMRI to investigate neuroplasticity in the primary sensory (S1) and motor cortex (M1) in patients with acute Bell's palsy (BP). We found that the period directly following the onset of BP (less than 14 days) is associated with significant decreases in regional homogeneity (ReHo), fractional amplitude of low frequency fluctuations (fALFF), and intrinsic connectivity contrast (ICC) values in the contralateral S1/M1 and in ReHo and ICC values in the ipsilateral S1/M1, compared to healthy controls. The regions with decreased ReHo, fALFF, and ICC values were in both the face and hand region of S1/M1 as indicated by resting-state fMRI but not task-related fMRI. Our results suggest that the early stages of BP are associated with functional neuroplasticity in both the face and hand regions of S1/M1 and that resting-state functional fMRI may be a sensitive tool to detect these early stages of plasticity in patient populations.

\section{Introduction}

Neuroplasticity is a common, yet complex phenomenon that occurs in both animals and humans [1-4]. In neural injury patients, this plasticity can play a significant role in the development of disease [5]. For example, following a stroke, the ipsilateral cortex becomes the dominant cortex used to control the affected limb, but with treatment there is a shift back to contralateral control [6, 7]. Amputation patients are another example of cortical map changes following a profound injury [8-10]. These neural injury models have provided investigators a chance to investigate neural plasticity in the human brain, and have significantly enhanced our understanding of the development of neural plasticity. However, the complexities of amputation and stroke studies, including variations in affected locations, duration of the injury, and accompanied psychiatric comorbidities, have limited the interpretation of the observed results.
Bell's palsy (BP) offers an ideal human model to investigate neuroplasticity following peripheral nerve injury [1120]. BP is the paralysis of the unilateral facial expression muscles caused by the reactivation of the herpes virus. This reactivation impairs the signaling from the motor cortex to the affected facial muscle. Compared to other conditions such as stroke and amputation, BP is associated with fewer complications and fewer or no medications, allowing us to explore the development of cortical reorganization directly following peripheral nerve injury.

The primary somatosensory cortex (S1) and the primary motor cortex (M1) are well known for their precise physical representation of the body on the cortex, thereby providing a perfect location to explore the reorganization of the brain caused by BP. Brain imaging tools such as MRI/fMRI, MEG, and PET and neuromodulation methods have allowed investigators to explore changes in the brain's structure, function, and excitability at $\mathrm{S} 1$ and $\mathrm{M} 1$ following different disorders [2127]. 


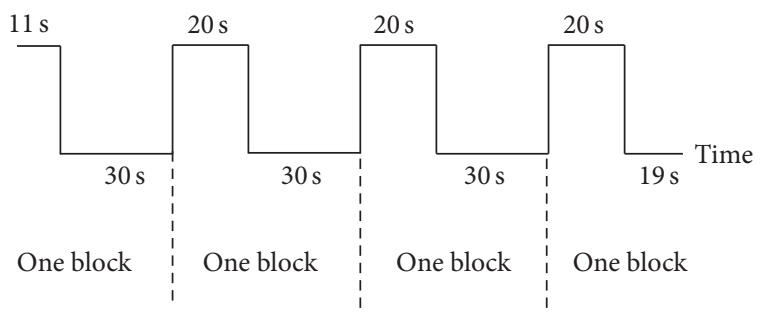

FIGURE 1: The block design paradigm used in this study.

Recently, intrinsic resting-state functional connectivity (rsFC) has drawn the attention of investigators, and been applied to investigate neural plasticity $[28,29]$. Different methods have been developed to investigate the restingstate functional connectivity. Some methods such as seedbased analysis and independent component analysis, focus on the networks changes (between/among different brain regions). Other methods, such as regional homogeneity (ReHo), intrinsic connectivity contrast (ICC), and fractional amplitude of low frequency fluctuations (fALFF), focus on regional brain functional status. $\mathrm{ReHo}$ is a measure of the time similarity of a given voxel to its nearest 26 voxels [30]. fALFF is used to characterize the regional, spontaneous neuronal activity $[31,32]$, while ICC tests the strength of the global connectivity pattern between each voxel and the rest of the brain [33]. Although each of these methods focus on different characteristics of resting-state functional connectivity, all of these methods may help us target key regions, rather than networks, providing valuable tools to study neuroplasticity in S1 and M1 in BP patients.

In this study we first used task-related fMRI (hand movement and mouth movement) to define subject's cortical map at $\mathrm{S} 1$ and $\mathrm{M} 1$ and then investigated and compared the regional brain functional status in the early period of BP to healthy controls using ReHo [30], fALFF [31, 32], and ICC [33]. Our objective was to find the acute functional influence of peripheral nerve injury on the central nervous system.

\section{Method}

2.1. Subjects. Twenty-five right-handed patients with left or right side Bell's palsy (House-Brackmann Scale (HBS) $\geq 3$, age $36 \pm 7$ years ranging from 23 to 50,15 males, 10 females, 14 right side facial paralysis) were recruited from the acupuncture department at the First Affiliated Hospital of Zhejiang Chinese Medical University. All patients with Bell's palsy onset less than 14 days underwent the MRI scan [11]. We recruited 25 age- and gender-matched healthy controls. No study participants had a history of physical or psychological disorders. The study was approved by the Ethics Committee at the First Affiliated Hospital of Zhejiang Chinese Medical University.

2.2. MRI Experimental Design. There were a total of 5 tasks in our design: a facial movement task, left and right hand movement tasks, and left and right facial sensory stimulation task. We used a block design in our 5 tasks (Figure 1). The frequency of the movement or sensory stimulation was 13-16 times per 20 seconds. In the facial movement task, subjects were asked to purse their lips. In the hand movement tasks, we asked subjects to open and close their right or left hand. In the facial sensory tasks, we brushed subjects' right or left jaw. Prior to being scanned, all participants underwent practice trials to ensure the mastery of these exercises. Each taskrelated fMRI scan contained 4 blocks. The instructions were explained orally. All patients and healthy controls finished the hand movement and facial sensory tasks, and 21 healthy controls finished the facial movement task. The BP patients were not required to perform the facial movements due to their inability to perform facial expressions.

2.3. MRI Imaging Acquisition. All scans were performed on the same 3.0 Tesla MR scanner (Magnetom Verio, Siemens, Germany) in order to obtain T1-weighted structural images and echo-planar $\mathrm{T} 2{ }^{*}$-weighted images (EPI). Structural images were obtained by MP-RAGE sequence: $\mathrm{TR}=1900 \mathrm{~ms}$, $\mathrm{TE}=2.45 \mathrm{~ms}, \mathrm{FA}=9$ degrees, voxel size $=1 * 1 * 1 \mathrm{~mm}$, matrix $=256 * 256$. Two hundred time points of functional restingstate data were acquired by EPI session: TR $=2000 \mathrm{~ms}$, TE $=30 \mathrm{~ms}, \mathrm{FA}=90$ degrees, slices $=33$, and voxel size $=4.0 \times$ $4.0 \times 4.0 \mathrm{~mm}$ at the beginning of the MRI scan, while subjects kept their eyes opened. Sixty time points of functional taskstate data were obtained by EPI sequence: TR $=3000 \mathrm{~ms}$, TE $=30 \mathrm{~ms}, \mathrm{FA}=90$ degrees, slices $=36$, voxel size $=3.2 \times 3.2 \times$ $3.0 \mathrm{~mm}$, and gap $=0.75 \mathrm{~mm}$.

2.4. fMRI Data Analysis. All functional data preprocessing and analyses were performed using Data Processing Assistant for Resting-State fMRI (DPARSF) software (http://rfmri.org/ DPARSF) [34] and CONN toolbox v15.g [35] (http://www.nitrc .org/projects/conn) in MATLAB (MathWorks, Inc., Natick, Massachusetts). DPARSF is based on Statistical Parametric Mapping (SPM8) (http://www.fil.ion.ucl.ac.uk/spm) and a Resting-State fMRI Data Analysis Toolkit (http://www .restfmri.net) [36]. All statistical analyses were performed by SPM8.

Before we processed the images, all BP patients with left side palsy and matched healthy controls were flipped along the $y$-axis (R-L flip) so their images could be directly compared to the patients with right side palsy [14]. Given the important role of $S 1$ and $\mathrm{M} 1$ in neural plasticity changes following a nerve injury $[10,37-41]$, we defined the bilateral pre- and postcentral gyrus as the region of interest (ROI), and made a mask based on automated anatomical labeling (AAL) [42].

2.5. Task Data Analysis. For each subject, all images were realigned using a six-parameter rigid-body transformation that corrected for motion artifacts. The images were then coregistered with the subjects' corresponding anatomical (T1weighted) images, normalized by using structural images unified segmentation, resampled to $3 \mathrm{~mm}$ cubic voxels, and spatially smoothed with a $6 \mathrm{~mm}$ full width at half maximum (FWHM) Gaussian kernel. 
A multiple regression analysis using a general linear model was performed to obtain statistical parametric maps calculated for all 5 conditions (facial movement, right/left side hand movement and right/left side facial sensory). Functional MRI signal time courses were high-pass filtered (128 s) and modeled as an experimental stimulus onset function, and convolved by the canonical hemodynamic response function (low-pass filter). Individual contrast images for each task were entered into a 2nd level random effects analysis to make inferences at the group level. For our one sample $t$ test comparing facial movement, hand movement, and facial sensory stimulation with baseline, a threshold of voxel-wise $p<0.05$ FWE corrected was applied. For between group comparisons (BP versus controls), we applied a threshold of $p<0.005$ and small volume FWE corrected $p<0.05$ in ROI regions as defined above. A threshold of voxel-wise $p<0.005$ uncorrected and cluster-level $p<0.05$ family wise error (FWE) correction was applied to non ROI brain regions.

2.6. Resting-State Data Analysis. For each subject, the first 10 volumes of functional data were discarded because of the signal equilibrium and subjects' adaptation to the imaging noise. The remaining volumes were then slice timing corrected, spatially realigned, and coregistered with the subject's corresponding anatomical (T1-weighted) images and segmented. Friston 24 head motion parameters $[43,44]$ and CSF signals were regressed out in ReHo and fALFF data. All images were normalized using structural unified segmentation and resampled to $3 \mathrm{~mm}$ cubic voxels, and then ReHo data was filtered using the low frequency band $(0.01-0.08 \mathrm{~Hz})$. Frames with FD $0.2 \mathrm{~mm}$ ("scrubbing") were removed. Individuals with $50 \%$ of their time series removed were excluded from the analyses. After scrubbing, no one was excluded from the analysis. We did a ReHo analysis by calculating the Kendall's coefficient of concordance (KCC) of a given voxel with those of its nearest neighbors (26 voxels) in a voxel-wise analysis [30]. The ReHo data was then spatially smoothed with a $6 \mathrm{~mm}$ FWHM Gaussian kernel.

fALFF data was smoothed after normalization, and then, temporal filtering was performed so that only the low frequency band (0.01-0.08) was examined in subsequent analyses of the low frequency fluctuations (LFF) amplitude.

After normalization, the ICC data underwent ART (http://www.nitrc.org/projects/artifact_detect) to eliminate correlations caused by head motion and artifacts, by identifying outlier time points in the motion parameters and global signal intensity. For each subject, we treated images as outliers if composite movement from a preceding image exceeded $0.5 \mathrm{~mm}$, or if the global mean intensity was $>3$ SDs from the mean image intensity for the entire resting scan. ICC analysis [33] was carried out by applying a voxelvoxel approach using the CONN toolbox v15.g. This analysis determined the strength of functional connectivity between each voxel and all other voxels in the brain. The ICC data was then smoothed with a $6 \mathrm{~mm}$ FWHM Gaussian kernel.

To explore the ReHo/fALFF/ICC differences between the BP patient group and healthy control group, a twosample $t$-test was performed on ReHo/fALFF/ICC $\mathrm{z}$-maps.
For between group comparisons (BP versus controls), we applied a threshold of $p<0.005$ and small volume FWE corrected $p<0.05$ in the ROI as defined above. A threshold of voxel-wise $p<0.005$ uncorrected and cluster-level $p<0.05$ family wise error (FWE) correction was applied to non ROI brain regions.

\section{Results}

3.1. Behavioral Results. All patients showed impairments in their left or right facial muscle function due to BP, with the HBS grades $\geq 3$. All MR scans were performed 3-14 days after BP onset (Table 1).

3.2. Task-Related fMRI Results. We found significantly increased activation at the bilateral S1/M1, supplementary motor area (SMA), and cerebellum in healthy controls during the facial movement task.

During the hand movement task, we found significantly increased activation at the contralateral S1/M1, thalamus, putamen, insula, bilateral SMA, and cerebellum in both BP patients and healthy controls. There was no significant difference between the two groups.

During facial sensory stimulation, we found significantly increased activation at the contralateral S1/M1, insula and S2 in both BP patients and healthy controls. We also found that in the healthy control group the (1) left facial sensory stimulation provoked significantly increased activation at the ipsilateral S1 and the (2) right facial sensory stimulation provoked significantly increases activation at the ipsilateral cerebellum (Table 2). There was no significant difference between the two groups.

3.3. Resting-State Functional Connectivity Results. Compared to healthy controls, BP patients showed (1) significant ReHo decreases in the bilateral S1/M1 and contralateral cingulate motor area (CMA), (2) significant fALFF decreases in the contralateral S1/M1, and (3) significant ICC decreases in the bilateral S1/M1 (Table 3, Figures 3 and 4).

To explore the association between the above measurements and the duration of the patients' Bell's palsy, we also performed regression analyses across all patients using SPSS 18.0 Software (SPSS Inc., Chicago, IL, USA), including age, gender, and HBS as covariates. We found a significant positive association between the ICC values of the contralateral S1/M1 and the duration of the patient's BP $(p=0.035)$ (Figure 2$)$.

\section{Discussion}

In this study, we explored neural plasticity in BP patients using task-related and resting-state fMRI. We found that the early stage of BP is associated with significant decreases in ReHo, fALFF and ICC values in the contralateral S1/M1, and with significant decreases in ReHo and ICC values in the ipsilateral S1/M1, compared to matched healthy controls. It is interesting to note that the regions with decreased ReHo, fALLFF and ICC values were not only in the cortical map representing the face, but also in the hand region of 
TABLE 1: Clinical data for all BP patients.

\begin{tabular}{|c|c|c|c|c|c|}
\hline Number & Gender & Age (years) & Paralyzed side & Duration (days) & HBS \\
\hline 1 & $M$ & 44 & $\mathrm{R}$ & 7 & 4 \\
\hline 2 & M & 42 & $\mathrm{R}$ & 10 & 3 \\
\hline 3 & M & 38 & $\mathrm{R}$ & 7 & 3 \\
\hline 4 & M & 25 & $\mathrm{~L}$ & 14 & 5 \\
\hline 5 & M & 39 & $\mathrm{R}$ & 7 & 4 \\
\hline 6 & M & 34 & $\mathrm{~L}$ & 9 & 4 \\
\hline 7 & $\mathrm{~F}$ & 34 & $\mathrm{R}$ & 5 & 4 \\
\hline 8 & $\mathrm{~F}$ & 34 & $\mathrm{R}$ & 6 & 5 \\
\hline 9 & $\mathrm{~F}$ & 23 & $\mathrm{R}$ & 5 & 4 \\
\hline 10 & M & 28 & $\mathrm{~L}$ & 8 & 5 \\
\hline 11 & M & 40 & $\mathrm{~L}$ & 8 & 5 \\
\hline 12 & M & 33 & $\mathrm{R}$ & 7 & 5 \\
\hline 13 & $\mathrm{~F}$ & 39 & $\mathrm{~L}$ & 4 & 5 \\
\hline 14 & M & 39 & $\mathrm{~L}$ & 6 & 5 \\
\hline 15 & $\mathrm{~F}$ & 45 & $\mathrm{~L}$ & 6 & 4 \\
\hline 16 & $\mathrm{~F}$ & 33 & $\mathrm{R}$ & 3 & 4 \\
\hline 17 & M & 26 & $\mathrm{~L}$ & 9 & 4 \\
\hline 18 & M & 26 & $\mathrm{R}$ & 10 & 4 \\
\hline 19 & M & 43 & $\mathrm{R}$ & 8 & 5 \\
\hline 20 & $\mathrm{~F}$ & 35 & $\mathrm{~L}$ & 8 & 6 \\
\hline 21 & $\mathrm{M}$ & 42 & $\mathrm{R}$ & 9 & 6 \\
\hline 22 & $\mathrm{M}$ & 31 & $\mathrm{R}$ & 5 & 6 \\
\hline 23 & $\mathrm{~F}$ & 50 & $\mathrm{~L}$ & 8 & 4 \\
\hline 24 & $\mathrm{~F}$ & 39 & $\mathrm{~L}$ & 8 & 6 \\
\hline 25 & $\mathrm{~F}$ & 34 & $\mathrm{R}$ & 5 & 4 \\
\hline
\end{tabular}

F, female; HBS, House-Brackmann Scale; L, left side; M, male; R, right side.

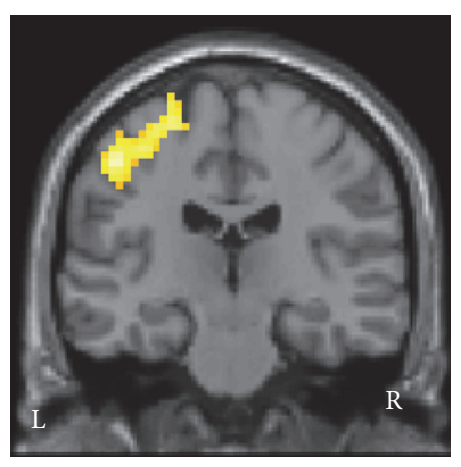

(a)
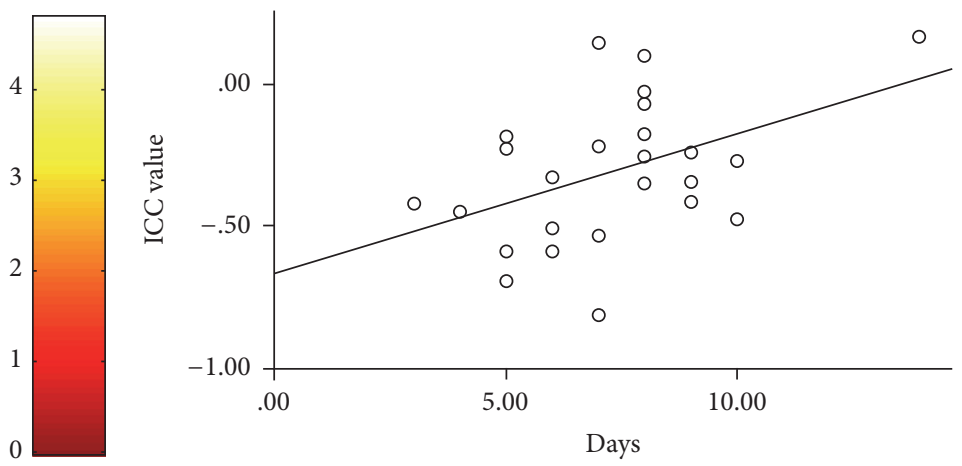

(b)

FIGURE 2: Intrinsic connectivity contrast (ICC) results. (a) ICC value was decreased in the contralateral S1/M1 in Bell's palsy patients. (b) There was a positive association between the duration of a subject's BP and the ICC value $(p<0.035)$.

the cortex; regions defined by hand and mouth task-related fMRI. We also found that BP patients and healthy controls had similar increased activation during the hand movement task and facial sensory stimulation. There was no significant difference between the two groups in all tasks performed by both groups. Our results suggest functional neuroplasticity in S1/M1 following the onset of BP and that resting-state fMRI may be a sensitive tool to detect these early stages of neural plasticity in patient populations.
ReHo, ICC, and fALFF are methods used to investigate the regional brain functional status using resting-state fMRI. ReHo measures the homogeneity of a brain's functional area in a specific condition, and has the ability to detect unpredicted hemodynamic responses that model-driven methods have failed to identify [30]. ICC tests the connectivity strength between each voxel and the rest of the brain [33]. fALFF measures regional, spontaneous neuronal activity, and can significantly suppress nonspecific signal components in 


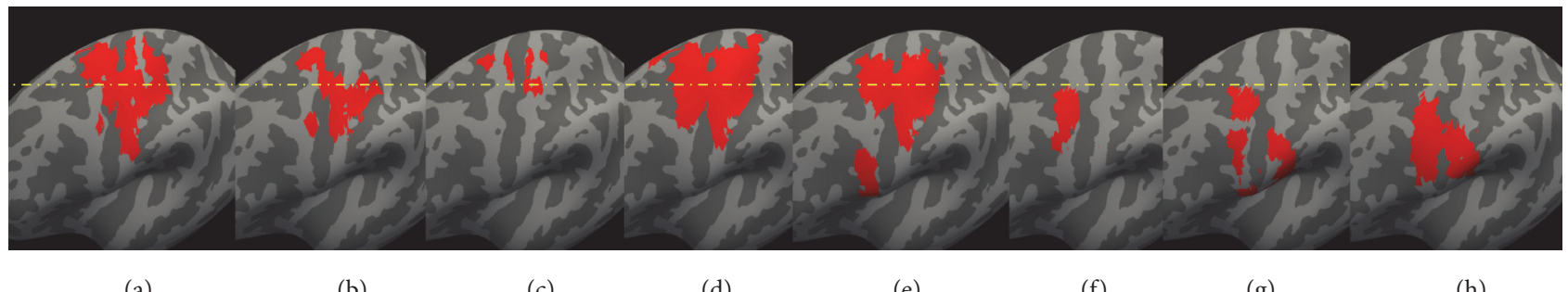

(a)

(b)

(c)

(d)

(e)

(f)

(g)

(h)

FIGURE 3: Contralateral hemisphere to the side of the affected nerve injury. (a) ReHo resting-state fMRI result. (b) ICC resting-state fMRI result. (c) fALFF resting-state fMRI result. (d) Increased activation evoked by ipsilateral hand movement in BP patients. (e) Increased activation evoked by ipsilateral hand movement in healthy controls. (f) Increased activation evoked by ipsilateral facial sensory stimulation in BP patients. (g) Increased activation evoked by ipsilateral facial sensory stimulation in healthy controls. (h) Increased activation evoked by mouth movement in healthy controls.

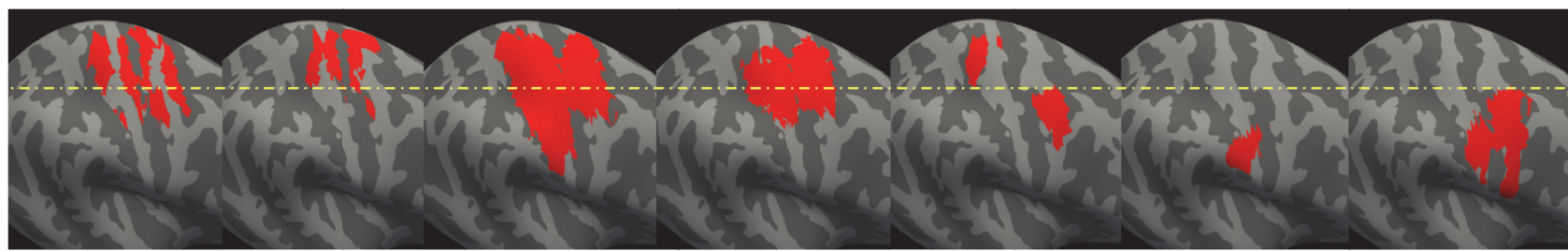

(a)

(b)

(c)

(d)

(e)

(f)

(g)

FIGURE 4: Ipsilateral hemisphere to the side of the affected nerve injury. (a) ReHo resting-state fMRI result. (b) ICC resting-state fMRI result. (c) Increased activation evoked by contralateral hand movement in BP patients. (d) Increased activation evoked by contralateral hand movement in healthy controls. (e) Increased activation evoked by contralateral facial sensory stimulation in BP patients. (f) Increased activation evoked by contralateral facial sensory stimulation in healthy controls. (g) Increased activation evoked by mouth movement in healthy controls.

TABLE 2: Task-related fMRI results for healthy controls.

\begin{tabular}{|c|c|c|c|c|c|c|}
\hline \multirow{2}{*}{ Tasks } & \multirow{2}{*}{ Brain region } & \multirow{2}{*}{ Cluster size } & \multirow{2}{*}{ Peak $Z$ score } & \multicolumn{3}{|c|}{ MNI coordinates $(\mathrm{mm})$} \\
\hline & & & & $x$ & $y$ & $z$ \\
\hline \multirow{4}{*}{ Facial movement } & L S1/M1 & 346 & 7.38 & -51 & -12 & 42 \\
\hline & R S1/M1 & 273 & 6.65 & 48 & -9 & 45 \\
\hline & R SMA & 175 & 6.42 & 6 & -3 & 63 \\
\hline & B cerebellum & 126 & 6.34 & 12 & -63 & -18 \\
\hline \multirow{3}{*}{ Right hand movement } & $\mathrm{L}$ S1/M1 & 1216 & 7.58 & -33 & -24 & $\overline{54}$ \\
\hline & B cerebellum & 505 & 7.27 & 12 & -54 & -15 \\
\hline & L thalamus & 535 & 7.01 & -15 & -21 & 6 \\
\hline \multirow{6}{*}{ Left hand movement } & R S1/M1 & 533 & 7.50 & 36 & -21 & 51 \\
\hline & L cerebellum & 552 & 7.14 & -18 & -54 & -18 \\
\hline & B SMA & 434 & 6.96 & 6 & 0 & 45 \\
\hline & $\mathrm{R}$ thalamus & 531 & 6.75 & 15 & -21 & 6 \\
\hline & R putamen & & 6.02 & 24 & 3 & 6 \\
\hline & R S2 & 149 & 6.05 & 48 & -21 & 24 \\
\hline \multirow{2}{*}{ Right facial sensory } & $\mathrm{L}$ S2/S1/M1 & 744 & 6.84 & -39 & -15 & $\overline{18}$ \\
\hline & $\mathrm{R}$ cerebellum & 42 & 5.84 & 6 & -66 & -12 \\
\hline \multirow{2}{*}{ Left facial sensory } & $\mathrm{R}$ S2/S1/M1 & 201 & 6.74 & 60 & -18 & 24 \\
\hline & L S1 & 29 & 5.47 & -60 & -18 & 30 \\
\hline
\end{tabular}

B, bilateral; L, left; M1, primary motor cortex; R, right; S1, primary somatosensory cortex; S2, secondary somatosensory cortex; SMA, supplementary motor area. 
TABLE 3: Comparisons of ReHo, fALFF, and ICC values in resting-state between BP patients and healthy controls using small volume FWE correction.

\begin{tabular}{|c|c|c|c|c|c|c|}
\hline \multirow{2}{*}{ Resting-state } & \multirow{2}{*}{ Brain region } & \multirow{2}{*}{ Cluster size } & \multirow{2}{*}{ Peak $Z$ score } & \multicolumn{3}{|c|}{ MNI coordinates $(\mathrm{mm})$} \\
\hline & & & & $x$ & $y$ & $z$ \\
\hline \multirow{2}{*}{ ReHo } & L S1/M1 & 500 & 4.59 & -33 & -24 & 63 \\
\hline & R S1/M1 & 234 & 4.09 & 24 & -36 & 60 \\
\hline fALFF & L S1/M1 & 40 & 3.36 & -30 & -27 & 63 \\
\hline \multirow{2}{*}{ ICC } & L S1/M1 & 224 & 4.33 & -48 & -18 & 48 \\
\hline & $\mathrm{R}$ S1/M1 & 140 & 4.60 & 15 & -21 & 69 \\
\hline
\end{tabular}

B, bilateral; L, left; M1, primary motor cortex; R, right; S1, primary somatosensory cortex.

resting-state MRI and increase sensitivity to regional spontaneous brain activity [32]. Although each of these methods focus on different aspects of brain function/activity, all of these methods focus on specific brain regions, rather than the networks, allowing us to target specific brain regions associated with the neuropathology of disorders.

In a previous study, investigators compared the restingstate functional connectivity of the motor network as identified by facial movements. They found that compared to healthy controls, BP patients showed decreased connectivity, mainly in areas responsible for sensorimotor integration and supervision (SII, insula, thalamus and cerebellum) [14]. In a more recent study, investigators found that the restingstate functional connectivity between the ipsilateral ACC and other brain regions such as the contralateral M1, SMA, ipsilateral S2, and mid-cingulate cortex increased with increased duration (ranging from 2-55 days) of BP [12]. In another study, investigators used the hand region of bilateral S1 as a seed to investigate the connectivity before and after acupuncture treatment at the contralateral side of the hand in BP patients. They found resting-state functional connectivity between S1 and bilateral S1, S2, and precuneus was significantly decreased in BP patients shortly following nerve injury [11].

In our study, we used methods focused on the regional brain status, and found significant decreases in resting-state functional connectivity at the S1/M1. This is consistent with previous studies on nerve injury. For instance, Pawela and colleagues found that the contralateral S1 showed a decreased connectivity with the bilateral M1 and ipsilateral S1 in rats with transected the brachial plexus compared to the control rats [45]. In another study, Makin and colleagues found the resting-state functional connectivity was decreased between the contralateral and ipsilateral hand regions in S1/M1 in upper-limb amputees compared to the healthy controls [22, 29].

We found decreased ReHo, fALFF, and ICC values not only at face representation areas, but also at hand representation areas of S1 and M1. These results are consistent with previous studies on neuroplasticity following peripheral nerve injury, in which researchers found the preservation of the original function of the invaded territory, as well as cohabitation with a newly acquired function $[29,46]$.

We also found a significant decrease in ReHo and ICC values at the ipsilateral S1/M1, suggesting that unilateral nerve injury can affect bilateral S1/M1. Studies have suggested that the ipsilateral hemisphere also plays an important role in sensorimotor function. For instance, a TMS study showed that stimulation to one side of the M1 cortex can cause ipsilateral motor evoked potentials (MEPs) in BP patients [20]. In amputation patients, imagining the movement of the phantom limb can evoke activation of bilateral S1/M1 $[9,47]$.

We found there was a significant positive association between BP duration and ICC values at the contralateral S1 and M1. We speculate this may reflect the self-recovery process of BP patients, which usually begins about one week after the onset of BP. Further studies are needed to validate the above findings.

In this study, we found that hand movement evoked significantly increased activation at bilateral M1, SMA, and insula, which is consistent with findings from previous studies $[14,15]$. In an early PET study on facial palsy patients, Rijntjes et al. found that finger tapping tasks provoked increased regional cerebral blood flow at the region of S1/M1 representing the hand and face in the all eight patients with facial palsy duration ranging from 29 days to 36 years compared to controls, but not in a patient with a duration of 7 days [18]. In another study, Klingner and colleagues found there was no significant difference in fMRI results between BP patients and healthy controls during a mouth movement task [14]. These results are consistent with our findings that there is not any significant difference in fMRI results between the acute stage of $\mathrm{BP}$ when patients are compared to healthy controls in the sensory and motor tasks.

The present study had several limitations. The first is that we did not scan the patients at late stage or after recovery, so we were not able to provide dynamic neural plasticity changes of BP patients. Second, we did not test the facial motor condition in our BP patients, therefore we were unable to measure the motor cortical function during this task and compare it to the healthy controls. However, a previous study found no significant difference between BP patients and healthy controls during a mouth movement task [14]. Finally, we used the ICC method, which measures both positive and negative functional connectivity. Positive and negative connectivity may be associated with different physiological meanings, but both represent valuable information. We would like to emphasize that this method has been successfully used in many other studies [48-53]. We 
therefore believe that ICC is an appropriate method to apply to this study.

\section{Conclusion}

We found that the period shortly following the onset of BP is associated with neural plasticity in both the face and hand region of the bilateral S1/M1 as measured by different regional functional connectivity methods. Our results imply that regional functional connectivity may be a useful tool to investigate neural plasticity in patient populations and may hold the potential to assess the severity of the injured nerve.

\section{Competing Interests}

The authors declare that they have no competing interests.

\section{Authors' Contributions}

Wenwen Song and Minhui Dai contributed equally to this work.

\section{Acknowledgments}

The study is supported by the Construction Funds of Key Subjects of Colleges and Universities in Zhejiang Province [Grant no. ZJGK2012-80-160]. Jian Kong is supported by R01AT006364, R01 AT008563, R21AT008707, and P01 AT006663 from NIH/NCCIH.

\section{References}

[1] J. H. Kaas, "Plasticity of sensory and motor maps in adult mammals," Annual Review of Neuroscience, vol. 14, pp. 137-167, 1991.

[2] J. A. Kleim, T. M. Hogg, P. M. VandenBerg, N. R. Cooper, R. Bruneau, and M. Remple, "Cortical Synaptogenesis and Motor Map Reorganization Occur during Late, but Not Early, Phase of Motor Skill Learning," Journal of Neuroscience, vol. 24, no. 3, pp. 628-633, 2004.

[3] R. J. Nudo and G. W. Milliken, "Reorganization of movement representations in primary motor cortex following focal ischemic infarcts in adult squirrel monkeys," Journal of Neurophysiology, vol. 75, no. 5, pp. 2144-2149, 1996.

[4] S. C. Cramer, "Repairing the human brain after stroke: I. Mechanisms of spontaneous recovery," Annals of Neurology, vol. 63, no. 3, pp. 272-287, 2008.

[5] S. Madhavan, L. M. Rogers, and J. W. Stinear, "A paradox: after stroke, the non-lesioned lower limb motor cortex may be maladaptive," European Journal of Neuroscience, vol. 32, no. 6, pp. 1032-1039, 2010.

[6] J. R. Carey, T. J. Kimberley, S. M. Lewis et al., "Analysis of fMRI and finger tracking training in subjects with chronic stroke," Brain, vol. 125, no. 4, pp. 773-788, 2002.

[7] S. C. Cramer, G. Nelles, R. R. Benson et al., "A functional MRI study of subjects recovered from hemiparetic stroke," Stroke, vol. 28, no. 12, pp. 2518-2527, 1997.

[8] H. Flor, L. Nikolajsen, and T. S. Jensen, "Phantom limb pain: a case of maladaptive CNS plasticity?" Nature Reviews Neuroscience, vol. 7, no. 11, pp. 873-881, 2006.
[9] K. MacIver, D. M. Lloyd, S. Kelly, N. Roberts, and T. Nurmikko, "Phantom limb pain, cortical reorganization and the therapeutic effect of mental imagery," Brain, vol. 131, no. 8, pp. 2181-2191, 2008.

[10] E. Raffin, N. Richard, P. Giraux, and K. T. Reilly, "Primary motor cortex changes after amputation correlate with phantom limb pain and the ability to move the phantom limb," NeuroImage, vol. 130, pp. 134-144, 2016.

[11] X. He, Y. Zhu, C. Li et al., "Acupuncture-induced changes in functional connectivity of the primary somatosensory cortex varied with pathological stages of Bell's palsy," NeuroReport, vol. 25, no. 14, pp. 1162-1168, 2014.

[12] S. Hu, Y. Wu, C. Li et al., "Increasing functional connectivity of the anterior cingulate cortex during the course of recovery from bell's palsy," NeuroReport, vol. 26, no. 1, pp. 6-12, 2015.

[13] C. M. Klingner, G. F. Volk, S. Brodoehl, H. P. Burmeister, O. W. Witte, and O. Guntinas-Lichius, "Time course of cortical plasticity after facial nerve palsy: a single-case study," Neurorehabilitation and Neural Repair, vol. 26, no. 2, pp. 197-203, 2012.

[14] C. M. Klingner, G. F. Volk, S. Brodoehl, O. W. Witte, and O. Guntinas-Lichius, "The effects of deefferentation without deafferentation on functional connectivity in patients with facial palsy," NeuroImage: Clinical, vol. 6, pp. 26-31, 2014.

[15] C. M. Klingner, G. F. Volk, A. Maertin et al., "Cortical reorganization in Bell's palsy," Restorative Neurology and Neuroscience, vol. 29, no. 3, pp. 203-214, 2011.

[16] S.-P. Kong, Q.-W. Tan, Y. Liu et al., "Specific Correlation between the Hegu Point (LI4) and the Orofacial Part: evidence from an fMRI Study," Evidence-based Complementary and Alternative Medicine, vol. 2015, Article ID 585493, 7 pages, 2015.

[17] C. Li, J. Yang, J. Sun et al., "Brain responses to acupuncture are probably dependent on the brain functional status," EvidenceBased Complementary and Alternative Medicine, vol. 2013, Article ID 175278, 14 pages, 2013.

[18] M. Rijntjes, M. Tegenthoff, J. Liepert et al., "Cortical reorganization in patients with facial palsy," Annals of Neurology, vol. 41, no. 5, pp. 621-630, 1997.

[19] A. Smit, J. van der Geest, M. Metselaar, A. van der Lugt, F. VanderWerf, and C. De Zeeuw, "Long-term changes in cerebellar activation during functional recovery from transient peripheral motor paralysis," Experimental Neurology, vol. 226, no. 1, pp. 33-39, 2010.

[20] S. Yildiz, F. Bademkiran, N. Yildiz, I. Aydogdu, B. Uludag, and C. Ertekin, "Facial motor cortex plasticity in patients with unilateral peripheral facial paralysis," NeuroRehabilitation, vol. 22, no. 2, pp. 133-140, 2007.

[21] G. Jiang, C. Li, J. Wu et al., "Progressive thinning of visual motion area in lower limb amputees," Frontiers in Human Neuroscience, vol. 10, article 79, 2016.

[22] T. R. Makin, N. Filippini, E. P. Duff, D. Henderson Slater, I. Tracey, and H. Johansen-Berg, "Network-level reorganisation of functional connectivity following arm amputation," NeuroImage, vol. 114, pp. 217-225, 2015.

[23] A. May and C. Gaser, "Magnetic resonance-based morphometry: a window into structural plasticity of the brain," Current Opinion in Neurology, vol. 19, no. 4, pp. 407-411, 2006.

[24] J. D. Meier, T. N. Aflalo, S. Kastner, and M. S. A. Graziano, "Complex organization of human primary motor cortex: a highresolution fMRI study," Journal of Neurophysiology, vol. 100, no. 4, pp. 1800-1812, 2008. 
[25] S. Preißler, J. Feiler, C. Dietrich, G. O. Hofmann, W. H. R. Miltner, and T. Weiss, "Gray matter changes following limb amputation with high and low intensities of phantom limb pain," Cerebral Cortex, vol. 23, no. 5, pp. 1038-1048, 2013.

[26] N. S. Ward, M. M. Brown, A. J. Thompson, and R. S. J. Frackowiak, "Neural correlates of motor recovery after stroke: a longitudinal fMRI study," Brain, vol. 126, no. 11, pp. 2476-2496, 2003.

[27] J. Kong, R. B. Spaeth, H.-Y. Wey et al., "S1 is associated with chronic low back pain: a functional and structural MRI study," Molecular Pain, vol. 9, article 43, 2013.

[28] G. Di Pino, G. Pellegrino, G. Assenza et al., "Modulation of brain plasticity in stroke: a novel model for neurorehabilitation," Nature Reviews Neurology, vol. 10, no. 10, pp. 597-608, 2014.

[29] T. R. Makin, J. Scholz, N. Filippini, D. Henderson Slater, I. Tracey, and H. Johansen-Berg, "Phantom pain is associated with preserved structure and function in the former hand area," Nature Communications, vol. 4, article 1570, 2013.

[30] Y. Zang, T. Jiang, Y. Lu, Y. He, and L. Tian, "Regional homogeneity approach to fMRI data analysis," NeuroImage, vol. 22, no. 1, pp. 394-400, 2004.

[31] Y. F. Zang, Y. He, C. Zhu et al., "Altered baseline brain activity in children with ADHD revealed by resting-state functional MRI," Brain \& Development, vol. 29, no. 2, pp. 83-91, 2007.

[32] X.-N. Zuo, A. Di Martino, C. Kelly et al., "The oscillating brain: complex and reliable," NeuroImage, vol. 49, no. 2, pp. 1432-1445, 2010.

[33] R. Martuzzi, R. Ramani, M. Qiu, X. Shen, X. Papademetris, and R. T. Constable, "A whole-brain voxel based measure of intrinsic connectivity contrast reveals local changes in tissue connectivity with anesthetic without a priori assumptions on thresholds or regions of interest," NeuroImage, vol. 58, no. 4, pp. 1044-1050, 2011.

[34] C.-G. Yan and Y.-F. Zang, "DPARSF: a MATLAB toolbox for 'pipeline' data analysis of resting-state fMRI," Frontiers in System Neuroscience, vol. 4, no. 13, 2010.

[35] S. Whitfield-Gabrieli and A. Nieto-Castanon, "Conn: a functional connectivity toolbox for correlated and anticorrelated brain networks," Brain Connect, vol. 2, no. 3, pp. 125-141, 2012.

[36] X.-W. Song, Z.-Y. Dong, X.-Y. Long et al., "REST: a toolkit for resting-state functional magnetic resonance imaging data processing," PLoS ONE, vol. 6, no. 9, Article ID e25031, 2011.

[37] M. Desmurget, F. Bonnetblanc, and H. Duffau, "Contrasting acute and slow-growing lesions: a new door to brain plasticity," Brain, vol. 130, no. 4, pp. 898-914, 2007.

[38] J. Doyon and H. Benali, "Reorganization and plasticity in the adult brain during learning of motor skills," Current Opinion in Neurobiology, vol. 15, no. 2, pp. 161-167, 2005.

[39] R. J. Nudo, E. J. Plautz, and S. B. Frost, "Role of adaptive plasticity in recovery of function after damage to motor cortex," Muscle and Nerve, vol. 24, no. 8, pp. 1000-1019, 2001.

[40] A. Pascual-Leone, A. Amedi, F. Fregni, and L. B. Merabet, "The plastic human brain cortex," Annual Review of Neuroscience, vol. 28, pp. 377-401, 2005.

[41] I. H. Robertson and J. M. J. Murre, "Rehabilitation of brain damage: brain plasticity and principles of guided recovery," Psychological Bulletin, vol. 125, no. 5, pp. 544-575, 1999.

[42] N. Tzourio-Mazoyer, B. Landeau, D. Papathanassiou et al., "Automated anatomical labeling of activations in SPM using a macroscopic anatomical parcellation of the MNI MRI singlesubject brain," NeuroImage, vol. 15, no. 1, pp. 273-289, 2002.
[43] K. J. Friston, S. Williams, R. Howard, R. S. J. Frackowiak, and R. Turner, "Movement-related effects in fMRI time-series," Magnetic Resonance in Medicine, vol. 35, no. 3, pp. 346-355, 1996.

[44] C.-G. Yan, B. Cheung, C. Kelly et al., "A comprehensive assessment of regional variation in the impact of head micromovements on functional connectomics," NeuroImage, vol. 76, pp. 183-201, 2013.

[45] C. P. Pawela, B. B. Biswal, A. G. Hudetz et al., "Interhemispheric neuroplasticity following limb deafferentation detected by resting-state functional connectivity magnetic resonance imaging (fcMRI) and functional magnetic resonance imaging (fMRI)," NeuroImage, vol. 49, no. 3, pp. 2467-2478, 2010.

[46] K. T. Reilly and A. Sirigu, "The motor cortex and its role in phantom limb phenomena," Neuroscientist, vol. 14, no. 2, pp. 195-202, 2008

[47] F. Hamzei, J. Liepert, C. Dettmers et al., "Structural and functional cortical abnormalities after upper limb amputation during childhood," NeuroReport, vol. 12, no. 5, pp. 957-962, 2001.

[48] A. Anticevic, M. S. Brumbaugh, A. M. Winkler et al., "Global prefrontal and fronto-amygdala dysconnectivity in bipolar I disorder with psychosis history," Biological Psychiatry, vol. 73, no. 6, pp. 565-573, 2013.

[49] A. Anticevic, S. Hu, S. Zhang et al., "Global resting-state functional magnetic resonance imaging analysis identifies frontal cortex, striatal, and cerebellar dysconnectivity in obsessivecompulsive disorder," Biological Psychiatry, vol. 75, no. 8, pp. 595-605, 2014

[50] M. Čeko, Y. Shir, J. A. Ouellet, M. A. Ware, L. S. Stone, and D. A. Seminowicz, "Partial recovery of abnormal insula and dorsolateral prefrontal connectivity to cognitive networks in chronic low back pain after treatment," Human Brain Mapping, vol. 36, no. 6, pp. 2075-2092, 2015.

[51] A. Demertzi, A. Van Ombergen, E. Tomilovskaya et al., "Cortical reorganization in an astronaut's brain after long-duration spaceflight," Brain Structure and Function, vol. 221, no. 5, pp. 2873-2876, 2016.

[52] W. R. Marchand, J. N. Lee, S. Johnson, P. Gale, and J. Thatcher, "Differences in functional connectivity in major depression versus bipolar II depression," Journal of Affective Disorders, vol. 150, no. 2, pp. 527-532, 2013.

[53] N. Yassi, C. B. Malpas, B. C. V. Campbell et al., "Contralesional thalamic surface atrophy and functional disconnection 3 months after ischemic stroke," Cerebrovascular Diseases, vol. 39, no. 3-4, pp. 232-241, 2015. 

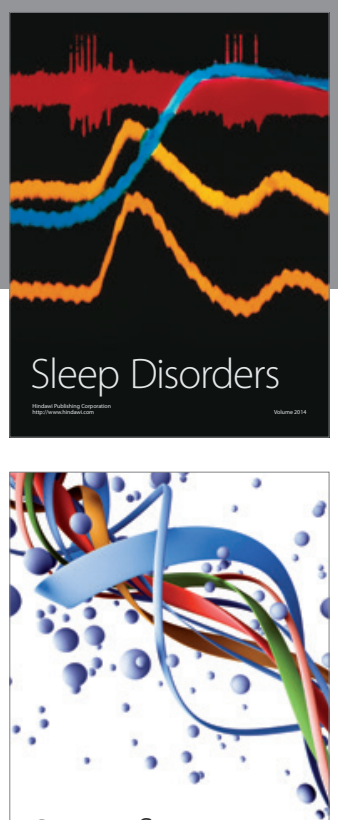

Scientifica
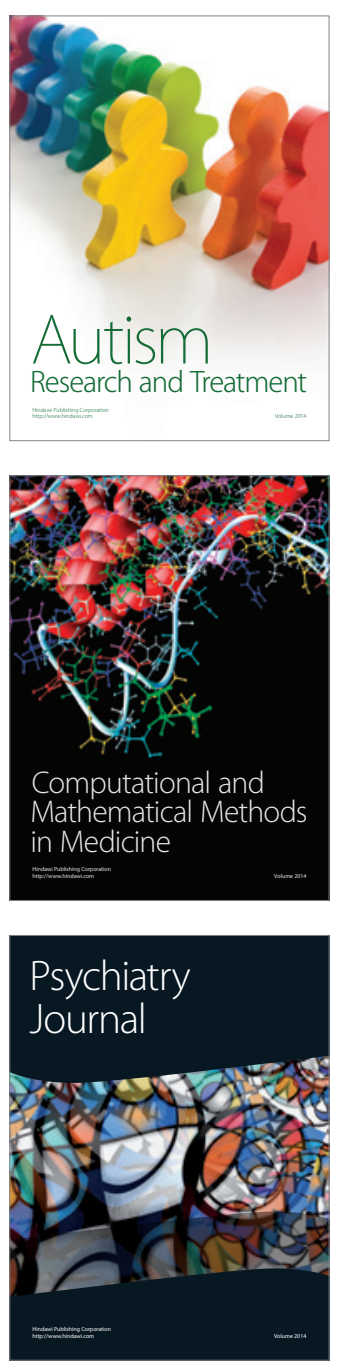
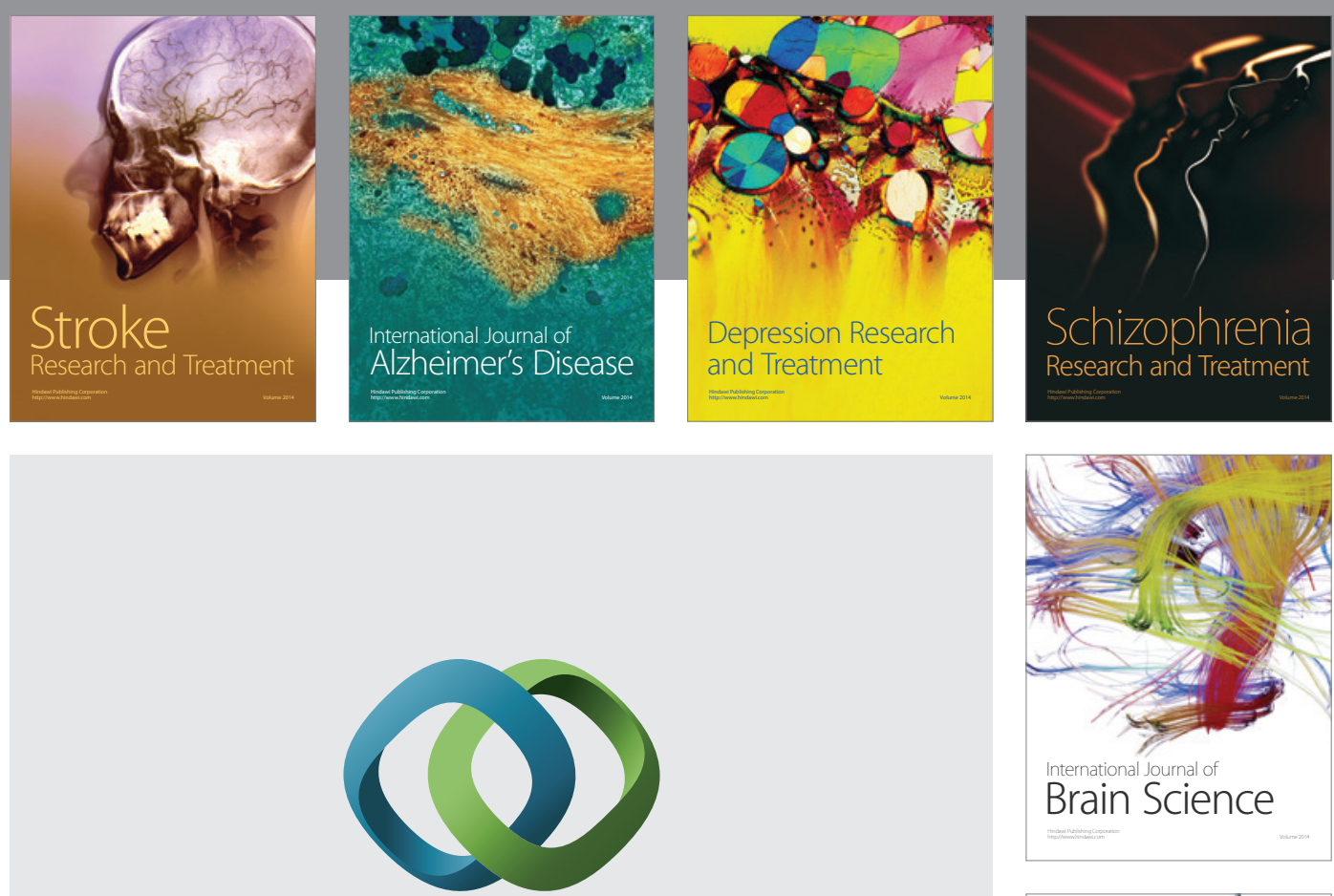

\section{Hindawi}

Submit your manuscripts at

https://www.hindawi.com
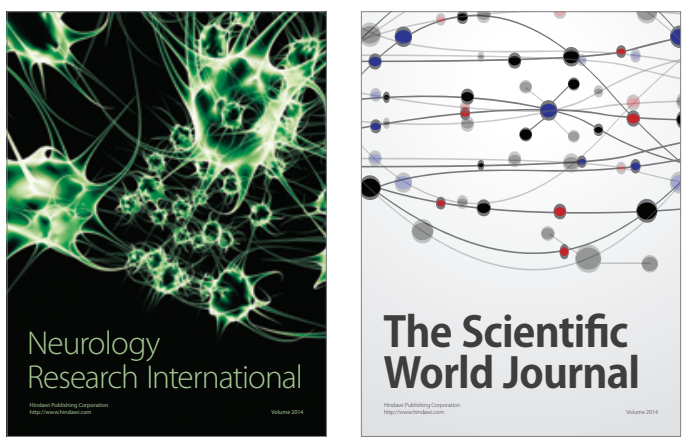

The Scientific World Journal





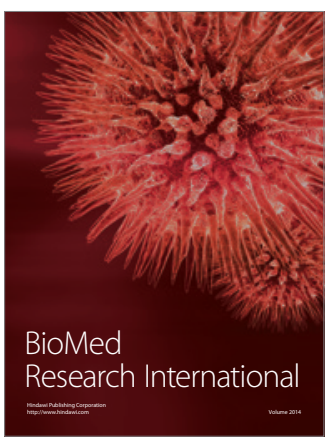

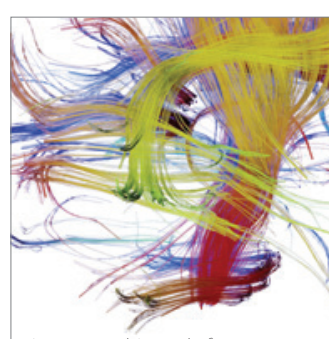

Brain Science



Neural Plasticity
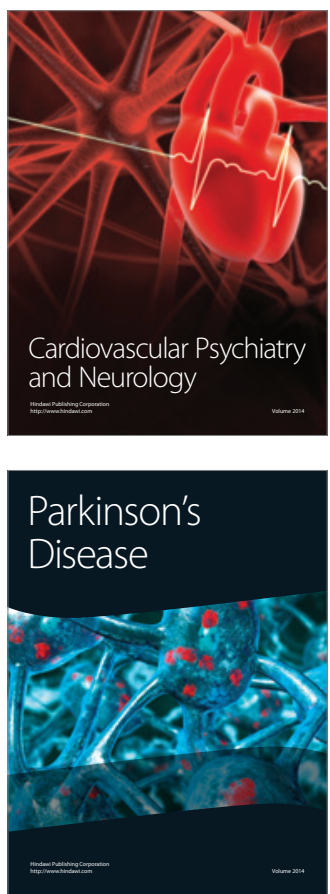